should become more and more intertwined. This book will help to accelerate the mixing process.

\title{
W. H. Gottschalk
}

Probability theory. By Michel Loève. 2d ed. Princeton, D. Van Nostrand Co., Inc. $16+685$ pp. $\$ 14.75$.

The second edition differs from the first principally in the addition of about 150 pages on continuous parameter stochastic processes. According to the first edition preface, calculus was a prerequisite. The second edition prerequisite is "honest" calculus. A student whose calculus course has been honest enough to prepare him for this difficult book is indeed fortunate. Even with his new section, Loève keeps to his original optimistic estimate that the material can be covered in about three to four semesters. However there is one concession: he simplifies at least his preface by warning the unlearned reader to be armed with "patience, pen, and calculus" instead of the first edition's "patience, pebble, and reed."

Loève's first edition marked the end of a golden research era in probability theory, in which the subject had been advancing too fast to be treated by anything but research articles, specialized or research books, and elementary texts. He was the first to attempt a text covering the most significant aspects of the subject in a rigorous manner. Even the over 600 pages of the second edition have of course necessarily omitted many things of interest, although the exceedingly compact style sometimes goes beyond letting the reader see beauty bare, in fact forcing him to guess at the beauty from the skeleton. But he has shown it possible to write a reasonably complete text as a central core, from which specialized works can radiate. His material includes far more than recent advanced texts by Fisz and Richter, written in a more leisurely style. Even though probability theory has reached the textbook stage, however, it seems premature to include as little history as Loève does. A bibliography is a poor substitute for frequent remarks on the background of the subject, including at least some dates if name-dropping is considered too hazardous.

Loève's new section has two chapters, both under the general heading "Elements of random analysis": XI Foundations; martingales and decomposability; XII Markov processes. "Foundations" contains a discussion of separability of processes, sample function continuity, and related topics. "Martingales" discusses martingales. "Decomposability" discusses what are called by various authors "integrals with independent elements," "additive processes," "differential processes," "processes with independent increments" and now 
by Loève "decomposable processes." This is a subject for which authors like to invent at least one new nomenclature. Loève makes a distinction between "random function" (a family of random variables) and "stochastic process" (never formally defined but, roughly, a class of random functions with common conditional distributions). This reader found the distinction, going back to ideas of Lévy, somewhat confusing, but perhaps a clearer discussion would make the distinction helpful. Dynkin has a careful definition along these lines in the Markov case. Chapter XII includes detailed discussions of the strong Markov property, sample function continuity, and semigroup analysis based on the work of Feller and Dynkin. This part of the subject is still under rapid development, and many readers will find Loève's treatment helpful as an introduction to material otherwise available only in papers scattered through the periodical literature. The relation to potential theory is not discussed.

This reader feels that Loève's attempt to be so complete in a book of normal length would have been more successful if about 100 more pages had been allotted, and devoted to discussion and examples, but the book is an excellent pioneering text which will have an enormous influence.

J. L. Doob

Vorlesungen über Orthogonalreihen. By F. G. Tricomi. Grundlehren der mathematischen Wissenschaften, Band LXXVI. SpringerVerlag, Berlin-Göttingen-Heidelberg, 1955. 8+264 pp. DM 37.60.

This book, like many of its author's other well-known books, originated in courses of lectures given at the University of Turin. It is an enlarged and considerably revised version of a preliminary (mimeographed) Italian edition (Serie Ortogonali di Funzioni, Gheroni, Torino, 1948) which is now out of print. The very competent translation is the work of Dr. F. Kasch, of Göttingen.

The author's aim is to provide a lucid, comparatively elementary, and highly readable introduction to orthogonal expansions, and in particular to trigonometric series and orthogonal polynomials. In this he succeeds admirably, demanding from the reader little more than a thorough knowledge of advanced calculus (some knowledge of the elementary theory of Lebesgue integrals, and perhaps a little more on infinite series than is contained in some advanced calculus courses). It is not part of the author's plan to replace Zygmund on trigonometrical series, or Szegö on orthogonal polynomials, to aim at encyclopedic completeness or at penetrating to the most modern parts of the theory; and he valiantly resists the temptation to enter 